\title{
Based on the Publish/Subscribe and RMI for the Railway Power Network Integrated Large Data Set Synchronization
}

\author{
Zhijian QU \\ School of Electrical and Automation Engineering \\ East China Jiaotong University \\ Nanchang, Jiangxi Province, China \\ e-mail: 08117324@bjtu.edu.cn
}

\author{
Ruilin ZHOU \\ School of Electrical and Automation Engineering \\ East China Jiaotong University \\ Nanchang, Jiangxi Province, China
}

\author{
Shengao YUAN \\ School of Electrical and Automation Engineering \\ East China Jiaotong University \\ Nanchang, Jiangxi Province, China
}

\begin{abstract}
Railway power network is an important part of modern rail transportation, especially in high-speed railway. There are three problems: (1) With the increasing number of high-speed rail mileage, along the line of signal equipment, lighting equipment and occlusion device increasing quickly; (2) As the high-speed rail traffic density continues to increase, the locomotive is constantly in the movement of the flow, resulting in the power grid, the number of primary load increases rapidly, in order to ensure the reliable power supply equipment, the need for real-time recording of these devices on-site operating data; (3) The railway power supply dispatching system can not be compatible, so the interaction is difficult that the information is difficult to share. In order to solve above problems, this paper proposes a new method of large data synchronization based on $P$ / S technology and RMI, which can reduce the coupling between a series of cooperative classes on the basis of improving system interaction efficiency and system compatibility Degree, easy to expand the system function, efficient completion of massive data across the database synchronization.
\end{abstract}

Keywords-railway power network; primary load; network structure; publishing / subscription technology; RMI

\section{RAILWAY POWER NETWORK}

Railway power network is a very important part of modern railway, which mainly includes railway traction power supply system and railway power supply system in two parts. Railway traction power supply system consists of traction substation, autotransformer and catenary and other components, its main role is through the catenary feed line for the electric locomotive and EMU rated voltage of $25 \mathrm{kV}$ single Phase power alternating power; railway power supply system consists of distribution, overhead lines and cables, etc., including the railway along the signal equipment, lighting equipment, power supply $10 \mathrm{kV}$ railway power lines and for the station and interval automatic blocking device power supply $10 \mathrm{kV}$ railway automatic Occlusion line[4]. In this paper, we establish a database for each systems, and then in the database for the corresponding system establish system table, the power supply departments according to their own functional needs to subscribe to different forms, as shown in Figure 1.

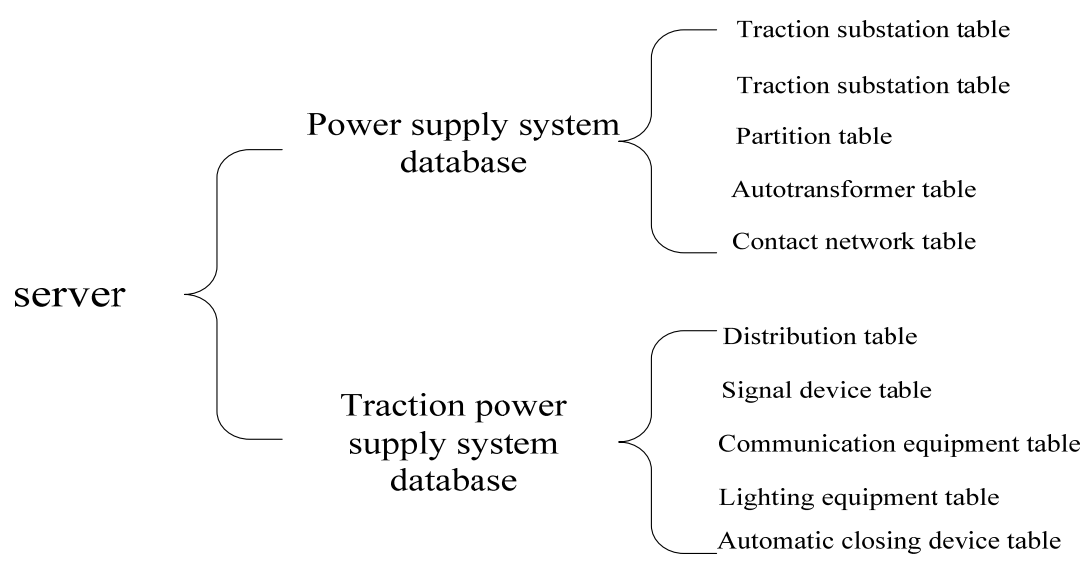

Figure 1. Power supply scheduling system table 
The railway catenary feed line and the power line need to be laid in full line, and the automatic blocking line is only laid in the automatic occlusion section and the station. In order to ensure the safe operation of the railway along the railway, the railway grid using dual power supply, dual-loop dual redundant backup power supply. The feeder is powered by traction substation at both ends, and the traction substation transforms the electric energy sent from the regional power system into the voltage and current level for

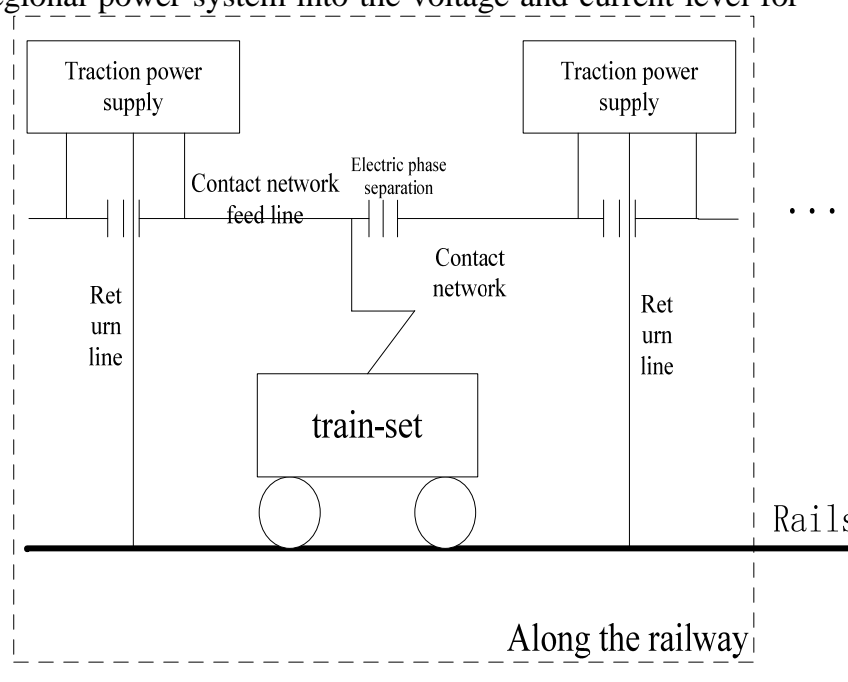

the traction electric locomotive and sends it to the feeder feedstock for the power locomotive. The line is powered by the two ends of the distribution, as shown in Figure 2, the distribution of A as the main supply, the distribution of B for the supply, when the line or self-closing line failure, the main supply A If the switch fails, the main supply center will try to close the switch again. If it fails again, use the low-side power supply automatic switching device of the through line and the self-closing line to switch the power supply.

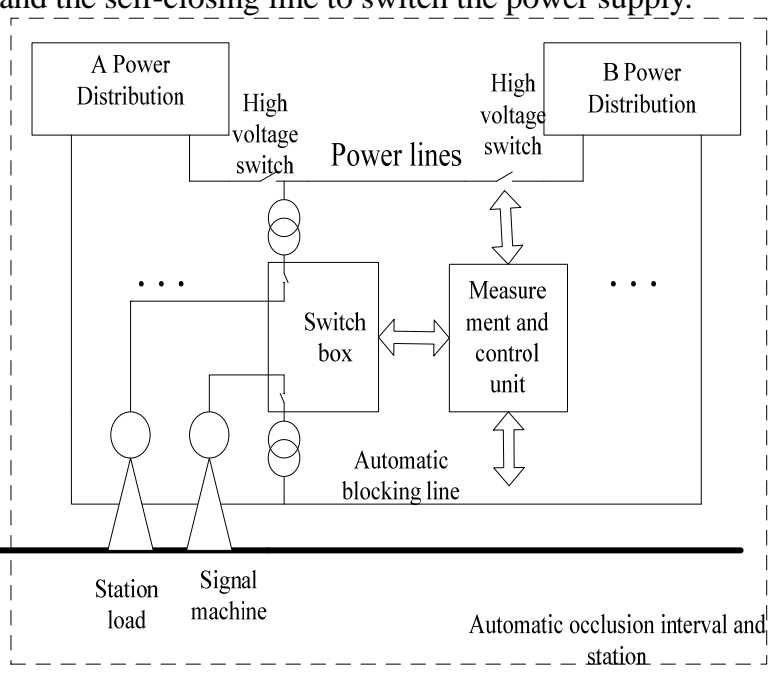

Figure 2. Railway grid structure

And a series of operations may lead to "blind control" phenomenon occurs, that is, after the failure of the railway power line failure, the fault information can not be synchronized to the railway power supply dispatch center, and the main supply A automatic switching standby power system can work properly, Will make the power supply department mistakenly believe that the power system is not a problem, when the backup power supply is also damaged after the repair is too late, may cause serious traffic accidents.

\section{PUBLISH/SUBSCRIBE MODEL}

Railway power dispatching system as a distributed system, involving tens of thousands of railway power supply departments, these units are located in China's large railway network along the various places, their location and behavior will affect or even change the entire system life cycle, In order to solve the problems caused by these constraints, we need a more flexible communication system. At present, the $2 \mathrm{~ms}$ polling information exchange mode adopted in the project also belongs to the traditional point-to-point synchronous communication mode, which will lead to a series of problems such as rigid and difficult system expansion. In order to maintain the consistency of things, when a power supply department database changes, the corresponding object also need to update the status of their own database, and the department did not know how many objects, specifically what objects need to make changes.

In order to solve the above problems, this paper proposes a large data synchronization method based on publish and subscribe. The publish subscribe model defines a one-to-many dependency that allows multiple subscribers to monitor a publisher and automatically notify subscribers when the publisher's own state changes so that they automatically update their status; on the other hand, The publish subscribe model can be interdependent several methods are individually packaged into different objects, so that they rely on the same abstraction, to provide large-scale distributed database required loosely coupled interactive way to complete the publisher and subscribers in the Time, space, synchronization on the complete decoupling[3], the specific structure shown in Figure 3.

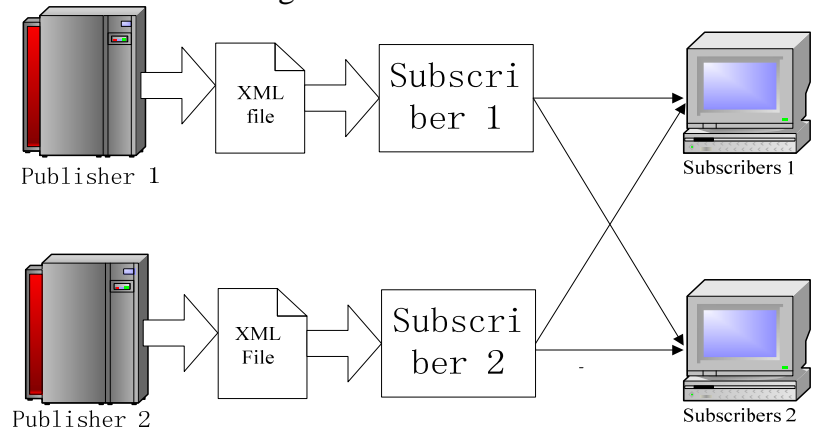

Figure 3. Releases the subscription decoupling mode

In this way, although the coupling between the publisher and the subscriber is solved, the coupling between the publisher and the subscriber is generated. For this reason, the 
decoupling method of the decorating mode is used, and the _Publication and _Subscription These two interfaces, the subscriber Replication Server class, inherit the two interfaces in order to implement bidirectional decoupling. In addition, the Publication class and the Subscription class inherit the _Publication and _Subscription interfaces, respectively, because the interface only defines Some abstract methods that do not have a method body itself have no other effect than other classes, so there is no possibility of coupling with other objects. The publisher publication class decouples with the subscriber ReplicationServer using the _Publication interface and through the internal encapsulation The publish () method pushes the message to the subscriber, and the Subscriber Subscription class decouples with the subscriber using the _subscription interface. The Subscription class can subscribe and drop messages through the internal subscribe () method and the unSubscribe () method. Set.

\section{REMOTE Method INVOKE}

Railway power monitoring data need to cross Different computer networks for transmission, and at the same time due to the existing computer network between the existing hardware and equipment operating system vary widely, so the development of cross-platform, portable, efficient and secure network distributed applications is particularly important in the current network development Commonly used to TCP / IP programming[1], which will naturally involve the use of Socket socket programming, and in complex distributed operating system using Socket socket programming requires a lot of repetitive coding, very troublesome, this article introduces the RMI technology will be a series of complex Socket development interface encapsulation in the bottom of the transport layer, the customer is completely transparent, to solve the system development complex and inefficient shortcomings.

RMI technology is a core solution for distributed application systems based on Java programming that can be deployed on any JRE-enabled platform with good cross-platform capability and enabling objects in different JVMs to look and behave Are the same as the local object, so you can call the remote object as a local object to use[2].

There are multiple publishers and subscribers in the railroad power dispatching system. Subscribers need to know which publishers exist in the system before ordering. In order to find the relevant subscriber subscription message, the subscriber needs to remotely call the target getRemotePublication () method to get the publisher information. The specific process shown in Figure 4.

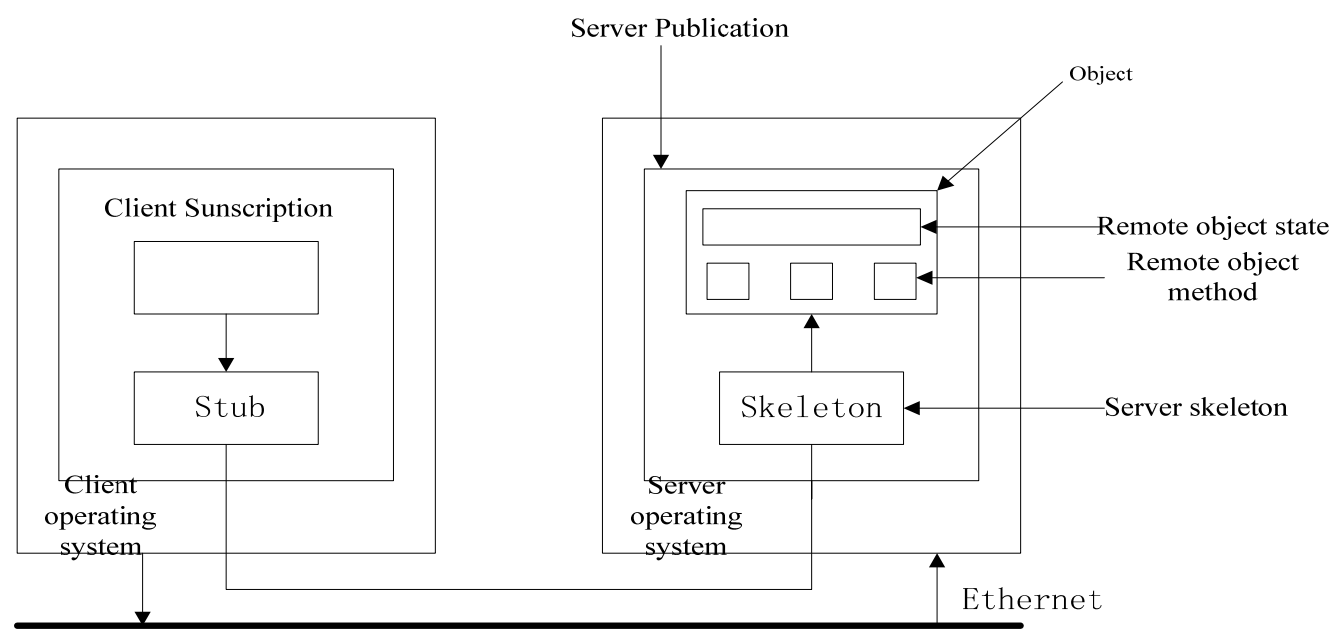

Figure 4. Remote method calls for distributed methods

Subscribers in the client, in order to get the publisher information in the server, access the server and look for the remote object Replication Server class that binds the getRemotePublication () method, the Replication Server class copies itself as a copy of the ReplicationServer_Stub after the socket socket Dynamically loaded into the client process as a local stub, the ReplicationServer_Stub proxy client communicates with the server skeleton ReplicationServer_Skeleton, requiring the skeleton to call the getRemotePublication () method in the ReplicationServer class to get information about all publishing services that exist on the server and deserialize the execution results And then to the ReplicationServer_Stub, where the subscriber has successfully obtained the publisher information.

\section{CONCLUSION}

In the server A establish a new database pub, we import the device telemetry information into table Railwayinfo that concludes 50000 volumes, in the server B establish a new blank database sub, the use of publish subscriptions + RMI large data remote new synchronization methods, the database sub successful subscription Pub, the results shown in Figure 5. 

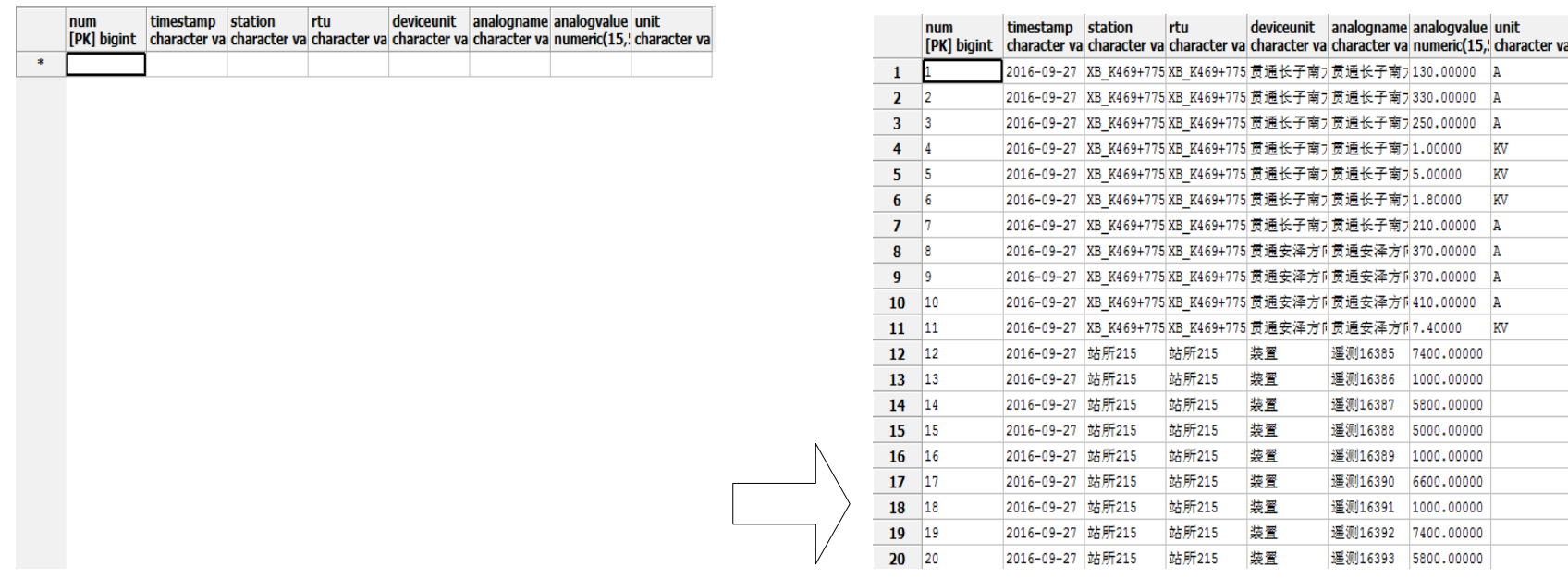

Figure 5. Experimental results

The results show that this method is effective in large data synchronization.

\section{ACKNOWLEDGEMENT}

This work is supported by National Natural Science Foundation of China (No. 51567008), Jiangxi Province Science Foundation for Distinguished Yong Scholars of China (No.20162BCB23045), Jiangxi Province Natural Science Foundation of China (No.20161BAB206156, No.20171BAB206044) and Jiangxi Province Educational Department Scientific Research Project (No.GJJ160471 ).

\section{REFERENCES}

[1] Zhao Zhuo, Zhao Xin, RMI distributed object technology research, microcomputer information, 2006,22 (3): 231-233.

[2] Liu Nan, Jin Wuping, RMI Distributed Object Technology Research and Application, Aeronautical Computing Technology, 2009,39 (1): 142-145.

[3] Wang Chen, publishing subscription system security key technology research, 2014, Beijing University of Posts and Telecommunications.

[4] Jin Haiqi, railway power information flow planning, 2006, Southwest Jiaotong University. 